\title{
Nachweis und Bestimmung geringster Nikotinkonzentrationen in Wasserproben
}

\author{
Von H. R. HEGI, ERIKA FISCHER, ERWIN MÄRKII)
}

Eidgenössische Anstalt für Wasserversorgung, Abwasserreinigung und Gewässerschutz an der Eidgenössischen Technischen Hochschule, Zürich

Direktor: Prof. Dr. O. Jaag.

Manuskript eingegangen am 26. Mai 1964

\section{Einleitung}

Gewässervergiftungen wurden schon in einigen Fällen mit grosser Wahrscheinlichkeit durch das Ablaufenlassen nikotinhaltiger Präparate (z. B. Tabaklauge) verursacht. 'Zur Abklärung der Ursache von Gewässervergiftungen spielt häufig die chemische Analyse eine entscheidende Rolle. Sie wird dadurch erschwert, dass sich vorerst über die als Ursache der Vergiftung in Frage kommenden Substanzen nur indirekt Vermutungen anstellen lassen, z. B. anhand der topographischen Lage von Gewerbeund Industriebetrieben. Zudem wird der Giftstoff im Zeitpunkt der Probenahme, die natürlich erst einige Zeit nach dem Auftreten der toxischen Schädigung, z. B. von Fischen, erfolgen kann, nur noch in sehr geringen Konzentrationen im Gewässer vorliegen.

Wir veröffentlichen nachstehend unsere methodischen Arbeiten, zu denen eine mutmasslich durch ein Nikotinpräparat verursachte Fischvergiftung den Anstoss gegeben hat. Die Arbeiten waren auf den möglichst sicheren qualitativen Nachweis und daneben auf die quantitative Bestimmung des Nikotins ausgerichtet. Von ganz besonderer Bedeutung war indessen die Notwendigkeit der Anreicherung des Nikotins aus Wasserproben, in denen es in extrem geringen Konzentrationen vorliegen kann.

Zur Anreicherung des Nikotins, das zur Bildung von Kationen befähigt

1) Für die praktische Mitarbeit sei Frl. M. Huber bestens gedankt. 
ist, haben wir die Anwendung eines Kationenaustauschers ausprobiert. Ionenaustauscher werden in der Analyse häufig zur Trennung von Stoffgemischen angewandt [I-3]; über deren Anwendung zur Anreicherung eines zu untersuchenden Stoffes sind hingegen bis jetzt in der Literatur noch wenig Angaben zu finden, obwohl sie zu diesem Zweck oftmals geeignet sein dürften. Zur Anreicherung und gleichzeitig zur Entfernung von Begleitstoffen eignet sich auch die bei Nikotinbestimmungen häufig angewandte Extraktion mit organischen Lösungsmitteln unter nachfolgender Reextraktion mit verdünnten Mineralsäuren [4]. Bei Wasserproben von mehreren Litern wäre die Anwendung des Extraktionsverfahrens mit organischen Lösungsmitteln, von denen grössere Mengen gebraucht würden, umständlich; das Ionenaustauschverfahren ist in einem solchen Falle besser zu handhaben. Ist aber das Volumen der Lösung, in der sich das Nikotin befindet, auf einige hundert Milliliter verringert worden, so eignen sich die Extraktion und Reextraktion zur weiteren Anreicherung sowie speziell zur Reinigung. Als weiteres Verfahren zur Reinigung nicht aber zur Anreicherung des Nikotins - eignet sich die Destillation aus alkalischer Lösung.

Zum eigentlichen Nachweis des Nikotins schien uns die spektrophotometrische Untersuchung im UV am ehesten geeignet [5-8]. Zur Bestätigung des Nachweises kann das Nikotin einer Farbreaktion unterworfen werden, welche ein im sichtbaren Bereich liegendes Absorptionsspektrum ergibt. Beide Methoden erlauben auch eine quantitative Bestimmung. Sollten diese Methoden zum sicheren Nikotinnachweis noch als ungenügend erachtet werden, so kann auch eine dünnschichtchromatographische Untersuchung angeschlossen werden [12], welche indessen als quantitative Methode ungenau ist. Weitere Methoden zur Nikotinbestimmung, wie z. B. die gravimetrischen und titrimetrischen Verfahren nach Fällung mit Kieselwolframsäure, Pikrinsäure oder dgl., die als quantitative Methoden wohl brauchbar sind, sollten in allen Fällen, wo die Spezifität des Nachweises im Vordergrund steht, wie z. B. bei der eingangs erwähnten Aufgabenstellung, nicht angewandt werden.

\section{Reagenzien}

\section{1 fïr Ionenaustausch, Extraktion und Destillation}

Nikotinreinsubstanz (von uns wurde Nicotinum purissimum «Siegfried» verwendet) zur Herstellung von Standardlösungen in o, $n n$-Schwefelsäure, 
Kationenaustauscher DOWEX so W, $\mathrm{X}_{4}$ ( $50 /$ roo mesh).

hergestellt aus konzentrierter SchweSchwefelsäure Io-volumprozentig felsäure p.a. (Vorsicht! In KunststoffSchwefelsäure etwa $0,1 n$ Schwefelsäure etwa 0,5 n

Ammoniak p.a. etwa 7,5prozentig. ampullen gelieferte Stammlösungen für eingestellte Schwefelsäure sind möglicherweise nicht spektralrein).

Petroläther, durch Ausschütteln mit Säure vorbehandelt: $500 \mathrm{ml}$ Petroläther Ph. Helv. V., mit dreimal $50 \mathrm{ml} \mathrm{o,I} n$-Schwefelsäure und $50 \mathrm{ml}$ Wasser schütteln.

Natriumhydroxyd p.a. fest, bzw. 32prozentige Lösung.

\section{2 für die Farbreaktion}

Puffer pH 6,5 nach Küster-Thiel-Fischbeck: II $876 \mathrm{~g} \mathrm{Na}_{2} \mathrm{HPO}_{4} \cdot 2 \mathrm{H}_{2} \mathrm{O}$ pro Liter und 9,078 $\mathrm{g} \mathrm{KH}_{2} \mathrm{PO}_{4}$ pro Liter. Von der Na-Salzlösung $3 \mathrm{I} 3 \mathrm{ml}$ und von der K-Salzlösung $687 \mathrm{ml}$ mischen.

Chloramin $\mathrm{I} \%$ : wässerige Lösung von p-Toluolsulfonchloramid-Na.

Kaliumzyanid, Iprozentige wässerige Lösung.

Barbitursäure, Iprozentige wässerige Lösung.

Natronlauge, etwa $0, \mathrm{I} n$ und etwa $2 n$.

\section{3 für die Dünnscbicbtcbromatographie}

Kieselgel G nach Stahl, für Dünnschichtchromatographie « Merck».

Kalilauge etwa $0,5 n$.

Chloroform Ph.H.V.

Äthanol 96prozentig.

Dragendorffreagens, weinsauer: $1,7 \mathrm{~g}$ Wismutsubnitrat werden in einer Lösung von $20 \mathrm{~g}$ Weinsäure in $80 \mathrm{ml}$ Wasser suspendiert. - $32 \mathrm{~g}$ Kaliumjodid werden in $80 \mathrm{ml}$ Wasser gelöst. Die beiden Lösungen werden gemischt. Bei Bedarf wird zu $5 \mathrm{ml}$ der Mischung eine Lösung von Io $\mathrm{g}$ Weinsäure in $50 \mathrm{ml}$ Wasser zugegeben.

\section{Geräte}

Chromatographierrohre mit Hahn, Rohr etwa I $50 \times 20 \mathrm{~mm}$.

Destillationsapparatur: Rundkolben mit Ansatz und Liebigkühler mit Vorstoss, Glasschliffverbindungen.

Spektralphotometer für UV und sichtbaren Bereich.

Grundausrüstung für Dünnschichtchromatographie. 


\section{Arbeitsvorschrift}

\subsection{Vorbereitung der Säulen}

Die Austauschermasse wird zuerst gewogen, dann über Nacht in entsalztem Wasser quellengelassen und ausgewaschen. 6 g DOWEX 50 W (gewogen in feuchtem Zustand, wie es aus der Originalpackung kommt, d. h. mit $65-71 \%$ Feuchtigkeit) werden in ein Chromatographierrohr auf eine Schicht Glaswolle eingefüllt. Bei einem Rohrdurchmesser von etwa $2 \mathrm{~cm}$ ergibt diese Austauschermenge eine Schichthöhe von ungefähr $6 \mathrm{~cm}$. Jede Säule wird dreimal mit $50 \mathrm{ml}$ 7,5prozentigem Ammoniak gewaschen, mit IoO $\mathrm{ml}$ Ioprozentiger Schwefelsäure regeneriert und mit entsalztem Wasser bis zur neutralen Reaktion gewaschen.

Eine Säule ist für höchstens 2,25 1 Wasserprobe zu verwenden. Falls man grössere Wassermengen aufarbeiten will, sind mehrere solche Säulen zu bereiten und Anteile von 2,25 1 durch je eine Säule durchlaufen zu lassen.

\subsection{Ionenaustausch}

Die Probe wird mit einer Geschwindigkeit von höchstens $30 \mathrm{ml}$ pro Minute durch die Säule geschickt. Diese enthält nun das Nikotin, welches mit 7,5prozentigem Ammoniak eluiert werden kann. Pro $6 \mathrm{~g}$ Austauscher verwendet man I50 ml Ammoniak, welches mit einer Geschwindigkeit von etwa $2 \mathrm{ml}$ pro Minute durchgeschickt wird.

Sollten die zu untersuchenden Proben mehr als etwa I mg Nikotin/l enthalten, so kann auf das Anreichern mittels Ionenaustauscher verzichtet werden.

\subsection{Extraktion}

Je $150 \mathrm{ml}$ ammoniakalisches Eluat werden mit $\mathrm{I}, 5 \mathrm{ml} \mathrm{32prozentiger}$ Natronlauge versetzt und mit viermal I $50 \mathrm{ml}$ Petroläther durch Ausschütteln im Scheidetrichter extrahiert. Die vereinigten Petrolätherauszüge $(600 \mathrm{ml})$ werden einmal mit $7 \mathrm{ml}$ und einmal mit $3 \mathrm{ml}$ entsalztem Wasser gewaschen und mit $20+20+10 \mathrm{ml} \mathrm{O,In}$ Schwefelsäure geschüttelt. Diese Säuremenge genügt auch für das doppelte Volumen Petroläther.

\subsection{Destillation}

Die vereinigten schwefelsauren Auszüge werden in einem etwa $200 \mathrm{ml}$ fassenden Destillationskölbchen mit $2 \mathrm{~g}$ Natriumbydroxyd p.a. alkalisch gemacht. Als Vorlage dienen $20 \mathrm{ml} \mathrm{o,5n} \mathrm{Schwefelsäure;} \mathrm{es} \mathrm{wird} \mathrm{mit} \mathrm{ein-}$ getauchtem Vorstoss destilliert. Wenn etwa $40 \mathrm{ml}$ übergegangen sind, wird die Destillation unterbrochen, nach dem Abkühlen dem Destillations- 
gut $20 \mathrm{ml}$ entsalztes Wasser zugefügt und es werden weitere $20 \mathrm{ml}$ überdestilliert. Das Destillat wird mit entsalztem Wasser auf Ioo $\mathrm{ml}$ ergänzt und enthält die gesamte Nikotinmenge der ursprünglichen Wasserprobe in $0, \mathbf{I} n$ Schwefelsäure.

\subsection{Spektrophotometrische Untersucbung im UV}

Vom Destillat ist im Spektralphotometer zwischen 215 und $280 \mathrm{~nm}$ eine Absorptionskurve aufzunehmen und mit einer Standardkurve in o, In Schwefelsäure (Nikotingehalt im Bereich desjenigen des zu untersuchenden Destillats) zu vergleichen. Der Verlauf der Kurve gibt ủber die Identität Auskunft, während aus dem Wert im Maximum bei $259 \mathrm{~nm}$ der Nikotingehalt der Probe berechnet werden kann.

Es dürfte im konkreten Falle dem Analytiker überlassen sein, zu entscheiden, ob die von WILLITS [6] sowie von WaLTZ [7] angegebene Korrektur zur Berechnung des Nikotingehaltes, basierend auf der Annahme eines geradlinigen Störspektrums, das von Begleitsubstanzen aus Tabak stammt, anzuwenden sei. Ein Störspektrum, stammend von im Wasser ubiquitär vorkommenden Stoffen, ist bei der Berechnung nicht zu berücksichtigen. Die Lösung ist anschliessend mit Ammoniak alkalisch $\mathrm{zu}$ machen und wiederum zwischen 215 und $280 \mathrm{~nm}$ zu spektrophotometrieren. Nikotin zeigt dabei die in Abschnitt 5.5 beschriebene leichte Verschiebung von Maximum und Minimum, wobei sich auch die Extinktion beim Maximum und Minimum in charakteristischer Weise ändert. Der Einfluss der Verdünnung durch die Ammoniakzugabe auf die Extinktion ist zu berücksichtigen.

\subsection{Farbreaktion und spektrophotometrische Untersuchung im sichtbaren Bereich}

Mit dem schwefelsauren Destillat kann eine weitere spektrophotometrische Untersuchung, basierend auf der Bildung eines Farbstoffes, vorgenommen werden. $50 \mathrm{ml}$ des Destillats, welche nach Möglichkeit zwischen $0, I$ und I mg Nikotin enthalten, werden zuerst mit $2 n$, dann mit $0, \mathrm{I} n$ Natronlauge auf einen $\mathrm{pH}$-Wert von $6,5-7$ gebracht und mit Puffer $\mathrm{pH} 6,5$ auf IOO $\mathrm{ml}$ aufgefüllt. Io $\mathrm{ml}$ davon werden mit je I $\mathrm{ml}$ Kaliumzyanid und Chloramin versetzt, während genau 5 min bei $25^{\circ}$ stehengelassen, dann mit einer Mischung von $20 \mathrm{ml}$ Puffer $\mathrm{pH} 6,5$ und ro $\mathrm{ml}$ Barbitursäure versetzt und mit Puffer $\mathrm{pH} 6,5$ auf $50 \mathrm{ml}$ ergänzt. Nach jedem Reagenszusatz soll gut gemischt werden. Nach einstündigem Stehenlassen bei $25^{\circ}$ wird gemessen. Die Nullprobe und diẹ Standardreihe werden wie die Proben behandelt; es ist hierbei auch von o,I $n$ 
schwefelsauren Lösungen auszugehen. Das mit Nikotin gebildete Reaktionsprodukt ist orangebraun. Im Spektralphotometer wird zwischen 450 und $550 \mathrm{~nm}$ eine Absorptionskurve aufgenommen, je nach dem Gehalt bei einer Schichtdicke von I bis $5 \mathrm{~cm}$. Die Kurve wird mit derjenigen, die mit Standardlösungen erhalten wird, in ihrem Verlauf qualitativ verglichen (Bestätigung des Nachweises). Daneben wird zur Berechnung des Nikotingehaltes die Extinktion beim Maximum ( $505 \mathrm{~nm}$ ) herangezogen.

\subsection{Dünnscbicbtcbromatograpbie}

Glasplatten sind mit einer Mischung aus $20 \mathrm{~g}$ Kieselgel G «Merck» und $40 \mathrm{ml} 0,5 n$ Kalilauge zu beschichten (zur Herstellung der Mischung während 30 Sekunden im Erlenmeyer mit Glasstopfen kräftig schütteln!). Die Platten sind Io min an der Luft, dann $30 \mathrm{~min}$ im Trockenschrank bei $100^{\circ} \mathrm{zu}$ trocknen.

Ein Teil des Destillats (s. Abschnitt 4.4) (z. B. 20 ml) wird mit Natronlauge alkalisch gemacht und viermal mit demselben Volumen (z. B. $20 \mathrm{ml}$ ) Petroläther extrahiert. Der Petrolätherauszug wird mit möglichst wenig Wasser gewaschen, mit entwässertem Natriumsulfat getrocknet und sorgfältig, am Schluss ohne zu erwärmen, so weit eingeengt, als zum Auftragen auf die Platten erforderlich ist.

Neben dem Petrolätherauszug werden auf weiteren Startflecken verschiedene Mengen von Nikotin, gelöst in Äthanol, aufgetragen (z. B. Io bis 5o $\gamma$ Nikotin). Lösungsmittel zum Chromatographieren: Chloroform/Äthanol 96prozentig, im Verhältnis 9: I. Das Lösungsmittel wird Io $\mathrm{cm}$ weit über die Startpunkte laufengelassen. Die Platten sind an der Luft zu trocknen und dann mit weinsaurem Dragendorffreagens zu besprühen. Nikotin gibt auf gelbem Grund einen bräunlichroten Fleck, Rf etwa 0,5 ; weniger als Io $\gamma$ Nikotinbase sind noch eindeutig wahrnehmbar.

\section{Eigene Versuche, Anmerkungen}

\subsection{Vorbereitung der Säule}

Die Säule muss zuerst mit Ammoniak gewaschen werden, weil sonst beim Eluieren des Nikotins im UV absorbierende Substanzen aus dem Austauscher herausgelöst werden.

\subsection{Ionenaustausch}

In Vorversuchen wurde durch Messungen im Spektralphotometer geprüft, unter welchen Bedingungen das Nikotin quantitativ von der 
Säule zurückgehalten werde. Falls die Säule so bereitet wurde, wie wir es in der Arbeitsvorschrift angegeben haben, wurde bei 3 I Leitungswasser, bzw. 2,5 1 Leitungswasser mit 10\% städtischem, vorgeklärtem Abwasser, noch kein Durchbruch des Nikotins festgestellt. Die Anwendung von 2,251 Wasserprobe dürfte deshalb unbedenklich sein; immerhin empfiehlt sich die spektrophotometrische Kontrolle einzelner Fraktionen des Durchlaufs.

Dann wurden Leitungswasserproben, welchen wir verschiedene Mengen Nikotin zugesetzt hatten, durch den Austauscher geschickt und in Anteilen von je so ml 7,5prozentigem Ammoniak eluiert. Das Nikotin konnte nahezu quantitativ in den Eluaten wiedergefunden werden, nämlich 96,5 bis 100\% des eingemessenen Nikotins.

\subsection{Extraktion}

Zuerst prüften wir den verwendeten Petroläther: Wir extrahierten $300 \mathrm{ml}$ mit dreimal $50 \mathrm{ml}$ o, I $n$ Schwefelsäure. Die Säurefraktionen wiesen im Bereich von $2 \mathrm{I} 5$ bis $280 \mathrm{~nm}$ von Fraktion zu Fraktion geringere Extinktionen auf; die dritte Fraktion war praktisch spektralrein.

Um das Nikotin möglichst vollständig als Base freizusetzen, wurden auf $100 \mathrm{ml}$ ammoniakalisches Eluat $\mathrm{I} \mathrm{ml} 32$ prozentige Natronlauge beigefügt.

Weiter wollten wir feststellen, welche Rolle die zur Extraktion verwendete Petroläthermenge spiele. Wir fügten auf $50 \mathrm{ml}$ Ammoniak $0, \mathrm{I} \mathrm{mg}$ und I mg Nikotin zu und schüttelten mit 50,75 , 100 und $200 \mathrm{ml}$ Petroläther aus. Die besten Werte resultierten, wenn das Verhältnis Ammoniak zu Petroläther (Gesamtmenge) I :4 gewählt wurde; es wurden hierbei 96, bzw. 93\% des eingemessenen Nikotins wiedergefunden, während die Ausbeute bei einem Verhältnis I:2 nur bei etwa $80 \%$ lag. Bei Anwendung von blossem Petroläther ist die Reextrahierbarkeit mit Säure sehr gross; ein Zusatz von Chloroform, der die Anwendung von weniger Petroläther ermöglichen würde, verringert sie beträchtlich.

\subsection{Destillation}

Durch die Destillation werden die aus dem Wasser stammenden, in das Extrakt gelangenden, geringfügigen Störspektren praktisch völlig beseitigt. Kontrolldestillationen zeigten, dass das Nikotin quantitativ übergeht; es wurden 100\% des eingemessenen Nikotins im Destillat wiedergefunden. 
Die Kombination der Ionenaustausch-, Extraktions- und Destillationsmethode wurde bei Proben aus Leitungswasser, dem wir 10\% vorgeklärtes städtisches Abwasser (durch Papierfilter filtriert) und Nikotin zugesetzt hatten, angewandt. Dabei wurden bei Zugabe von I mg Nikotin auf 51 Probe $83 \%$ des Nikotins wiedergefunden. Die Absorptionskurve war mit derjenigen von reinem Nikotin praktisch identisch.

\subsection{Spektrophotometrische Untersuchung im $U V$}

Es wurden mehrere Spektren von Nikotinstandardlösungen in $\mathrm{O}, \mathrm{I} n$ Schwefelsäure, ferner in 7,5prozentigem Ammoniak aufgenommen. Im sauren sowie im alkalischen Milieu lagen Maxima und Minima bei den in der Literatur [5-8] angegebenen Wellenlängen. Bei ıo mg Nikotin/l und einer Schichtdicke von 1o $\mathrm{mm}$ betrug die Extinktion in der schwefelsauren Lösung beim Maximum (259 nm) o,323, beim Minimum ( $226 \mathrm{~nm}$ ) 0,013 , in der ammoniakalischen Lösung beim Maximum (26I nm) o,I89 und beim Minimum (229 nm) 0,057. Oberhalb $280 \mathrm{~nm}$ absorbiert Nikotin weder in saurer noch in alkalischer Lösung. Die Messungen wurden mit dem Zeiss-Spektralphotometer PMQ II durchgeführt. Auch der Verlauf der Kurven entsprach den in den erwähnten Publikationen enthaltenen Angaben und graphischen Darstellungen.

Um festzustellen, ob aus Wasser, bzw. städtischem Abwasser, bei der vorgeschriebenen Aufarbeitung ubiquitäre Störsubstanzen auftreten, wurde eine Probe mit 10\% städtischem Abwasser und 90\% Leitungswasser ohne Nikotinzugabe extrahiert und destilliert. Das in $0, \mathrm{I} n$ Schwefelsäure aufgenommene Destillat absorbierte im Bereich von 2IS bis $280 \mathrm{~nm}$ praktisch nicht, während im schwefelsauren Auszug des Petrolätherextraktes (vor der Destillation) noch eine über einen grossen Wellenlängenbereich ziemlich gleichmässige Extinktion (um 0,02 bei einer Schichtdicke von Io $\mathrm{mm}$ ) gemessen worden war. Die spektrophotometrische Untersuchung im UV wird also durch normale Wasser- und Abwasserkomponenten bei der vorgeschriebenen Aufarbeitung der Proben praktisch nicht gestört.

Es interessierte uns ferner, ob störende Substanzen aus einem Tabakextrakt (v.a. Nebenalkaloide), welche mehr oder weniger in handelsübliche Nikotinpräparate gelangen können, den Verlauf der Absorptionskurve erheblich ändern. I $\mathrm{g}$ Tabak wurde mit dreimal $50 \mathrm{ml}$ o, In Schwefelsäure während Is min unter leichtem Erwärmen extrahiert. Die vereinigten Extrakte wurden mit $2 \mathrm{~g}$ Natriumhydroxyd alkalisch gemacht und wie in der Arbeitsvorschrift beschrieben destilliert. Der Verlauf der 
zwischen 215 und $280 \mathrm{~nm}$ aufgenommenen Kurve entsprach annähernd demjenigen von reinem Nikotin in O, In Schwefelsäure.

\subsection{Farbreaktion und spektrophotometrische Untersucbung im sichtbaren Bereich}

Der Bestimmung liegt folgende Farbreaktion zugrunde: Bei der Einwirkung von Brom- oder Chlorzyan auf Pyridinderivate entstehen bei Anwesenheit primärer aromatischer Amine (oder verwandter Stoffe, z. B. Barbitursäuren) farbige Schiffsche Basen des Glutaconaldehyds (Polymethinfarbstoffe) [9].

Wir änderten die Vorschrift von Asmus [ro] insofern ab, als wir von $0, \mathrm{I} n$-schwefelsauren Lösungen ausgingen, weil die Bestimmung mit dem Destillat, das nach der in Abschnitt 4.4 angegebenen Vorschrift erhalten wird, auszuführen ist. $50 \mathrm{ml}$ Destillat wurden neutralisiert und mit Puffer $\mathrm{pH}$ 6,5 auf Ioo $\mathrm{ml}$ aufgefüllt. Geprüft wurden Standardreihen mit o,OI-O,I mg Nikotin in Io $\mathrm{ml}$ mit Puffer versetzter Lösung. Da nur die Werte gleichzeitig angesetzter Reihen schöne Übereinstimmung zeigten, ist auf die in der Photometrie allgemein übliche Forderung, dass Standardreihen und Proben gleichzeitig angesetzt werden, speziell Gewicht zu legen.

Wenn die Io $\mathrm{ml}$ gepufferter Lösung (entsprechend $5 \mathrm{ml}$ Destillat), welche für die Farbreaktion verwendet wurden, o, I mg Nikotin enthielten (=20 mg Nikotin/l Destillat), stellten wir bei einer Schichtdicke von Io $\mathrm{mm}$ bei $505 \mathrm{~nm}$ eine durchschnittliche Extinktion von 0,35 fest. Die Farbreaktion ist also ungefähr halb so empfindlich wie die spektrophotometrische Bestimmung im UV in saurer Lösung.

\subsection{Spezifität des vorgeschriebenen Verfahrens}

Pyridin und manche Pyridinderivate, speziell mit Nikotin nahe verwandte Stoffe, weisen im UV ähnliche Absorptionsspektren auf wie das Nikotin. Deshalb schreiben wir die möglichst genaue Aufnahme der Absorptionsspektren vor; falls sie mit denjenigen des Nikotins praktisch identisch sind, so dürfte mit grosser Wahrscheinlichkeit auf die Anwesenheit von Nikotin oder Tabakextrakten zu schliessen sein.

Auch die Farbreaktion (s. Abschnitt 5.6) wird von Pyridin und manchen seiner Derivate gegeben. Die Spektren der gebildeten Farbstoffe dürften aber doch eine teilweise Differenzierung erlauben; Asmus $[9, \mathrm{ro}]$ beschreibt z. B. den Unterschied der Absorptionskurven der aus Pyridin und Nikotin gebildeten Farbstoffe. 


\subsection{Empfindlicbkeit des vorgeschriebenen Verfabrens}

Bei der Aufarbeitung nach unserer Vorschrift ist das in 2,25 1 Probe enthaltene Nikotin nach dem Ionenaustausch in $150 \mathrm{ml}$ Eluat enthalten. Dadurch wird die Lösung I smal konzentrierter. Durch die nach der Extraktion des Eluats mit Petroläther erfolgte Reextraktion mit gesamthaft $50 \mathrm{ml}$, I $n$ Schwefelsäure wird die Konzentration wiederum dreimal erhöht, während durch die Destillation wieder eine Verdünnung I:2 erfolgt. Das Destillat ist somit an Nikotin 22,5mal konzentrierter als die Probe. Im Destillat ist bei einer Schichtdicke von $50 \mathrm{~mm}$ zur Erreichung einer Extinktion von 0,I (beim Maximum $259 \mathrm{~nm}$ ), welche wir für den Nachweis von Nikotin anhand der Absorptionskurve im UV als untere Grenze annehmen möchten, eine Konzentration von etwa 0,6 mg Nikotin/1 Messlösung, d. h. von etwa 0,027 mg Nikotin/1 Probe erforderlich. Natürlich kann man eine weitergehendere Anreicherung vornehmen, indem man ein grösseres Probenvolumen anwendet und z. B Extraktion und Reextraktion wiederholt. Es dürfte auf diese Weise möglich sein, Nikotin in der Konzentration von wenigen $\gamma / 1$ Probe nachzuweisen.

\section{Zusammenfassung}

Es wird ein Verfahren zum Nachweis und zur Bestimmung von Nikotin vorgeschlagen, dessen Anwendung für die Abklärung der Ursache von Gewässervergiftungen geeignet sein dürfte. Daneben dürfte es zur Untersuchung von Wasserproben auf Nikotin in allen andern Fällen zu empfehlen sein, eventuell unter Vornahme von Vereinfachungen bei der Aufarbeitung. Zur Aufarbeitung der Proben nach unserer Vorschrift werden nacheinander das Kationenaustausch-, Extraktions- und Destillationsverfahren angewandt, zum Nachweis und zur Bestimmung die Spektrophotometrie und die Dünnschichtchromatographie.

\section{LITERATURVERZEICHNIS}

\section{Ionenaustausch}

[I] O Samuelson, Ion Exchangers in Analytical Cbemistry (Wiley \& Sons, New York I952).

[2] K. DORFNER, Ionenaustauscher (W. de Gruyter \& Co., Berlin I963).

[3] E. Haeberli, Pharmaceut. Acta Helv. 34, 65 (1959).

Extraktion, Destillation

[4] A. Wenusch, Z. Unters. Lebensm. 67, 601 (1934). 
Spektrophotometrie im $U V$

[5] M. Swain, J. Amer. Chem. Soc. 71, I34r (1949).

[6] C. O. Willits, Anal. Chemistry 22, 430 (I950).

[7] P. Waltz, Mitt. Geb. Lebensm'unters. u. Hyg. 50, I 59 (I959).

[8] K. Eschle, Mitt. Geb. Lebensm'unters. u. Hyg. 50, 258 (I959).

Farbreaktion und Spektrophotometrie im Sicbtbaren

[9] F. FEIGL, Tüpfelanalyse: II. organischer Teil, S. 283 (Akad. Verlagsges., Frankfurt a. M., 4. Aufl., I960).

[ro] E. Asmus, Z. anal. Chem. 185, 201 (1962).

[II] E. Asmus, Z. anal. Chem. 187, 33 (I962).

\section{Dünnscbicbtchromatograpbie}

[12] K. Randerath, Dünnschicbtcbromatographie (Verl. Chemie, Weinheim, 1962).

Separatdruck aus der Schweiz. Z. Hydrologie 26 , Fasc. I, I964. Herausgegeben mit Unterstützung der Stiftung der Wirtschaft zur Förderung des Gewässerschutzes in der Schweiz. 